\title{
A Rare case of Pleuro-Pulmonary Blastoma in A 2-Year-old Boy: the value of multi Modality Imaging in Diagnosis And Management.
}

\author{
Idris S.K ${ }^{1}$.,Osawe A.A ${ }^{1}$.,Saleh M.K ${ }^{1}$. \\ ${ }^{I}$ Department of Radiology Bayero University/Aminu Kano Teaching Hospital, Kano-Nigeria.
}

\begin{abstract}
This is a case of a 2 year old boy who presented with a history of sudden onset but progressively worsening symptoms of cough, respiratory difficulty and wheezing for 4 weeks. Chest X-ray revealed nearly complete opacity of the right hemithorax, while axial and coronal C.T. Scan images confirmed the presence of a huge extensive right sided hypodense (solid) intrapulmonary mass displacing the mediastinum and compressing the left lung. Histology following incisional biopsy confirmed a type III, pleuro- pulmonary blastoma.
\end{abstract}

\section{Introduction}

Blastomas are tumors virtually unique to childhood. They are thought to arise from immature, primitive tissues that present persistent embryonal elements on histology. These tumors affect younger pediatric population and are usually malignant ${ }^{1}$. Pleuropulmonary blastoma (PPB) is a rare entity with incidence between 0.25 to $0.5 \%$ of all primary lung malignancies that occur exclusively in children younger than 6 years and have a slight male preponderance $(1.5 / 1)^{2}$. The tumor usually originates from pulmonary parenchyma but may also arise from the mediastinum, diaphragm and/or pleura ${ }^{3}$. This has raised the possibility that PPB might originate from the splanchno-pleural or somato-pleural mesoderm. Although the exact etiology of PPB remains unknown, pathogenetic molecular abnormalities have been reported ${ }^{1}$

Initial symptoms and signs imitate a respiratory tract infection with or without fever, accompanied by mild to moderate respiratory distress ${ }^{4}$. Imaging plays an important role in the identification, management and follow-up of patients with this rare childhood malignancy ${ }^{5}$. The rarity of this tumor and its ability to mimic various chest infections especially the pneumonias prompted the report of this case.

\section{Case Report}

I.M. is a two-year-old boy who presented to the pediatric out patient department of Aminu Kano Teaching Hospital on account of cough, respiratory difficulty and wheezing for 4 weeks. Symptoms began suddenly and progressively worsened in the last 2 weeks prior to presentation. He had no history to suggest a recent respiratory viral infection, foreign body aspiration or family history of allergy. Physical examination revealed an acutely ill looking boy in respiratory distress, febrile (temperature of $37.8^{\circ} \mathrm{C}$ ) but not pale. His respiratory rate was 60 cycles/min and he had audible wheeze. Lung auscultation revealed decreased breath sounds in the right hemithorax with dull percussion notes over same area. His pulse rate was $108 / \mathrm{min}$. Other systems examination were unremarkable. Laboratory investigations showed leucocyte count of $19,400 / \mathrm{mm}^{3}$, hemoglobin $11.2 \mathrm{~g} / \mathrm{dL}$, platelet count $540,000 / \mathrm{mm}^{3}$, erythrocyte sedimentation rate $80 \mathrm{~mm} / \mathrm{h}$. Admission chest radiograph showed a huge mass of soft tissue density in the right hemithorax causing mild mediastinal shift to the left (Fig 1). However no obvious tracheal displacement or pleural effusion was seen. The adjacent rib cage was intact.Computed tomography (CT) of the thorax revealed an extensive, minimally enhancing, hypodense mass (HU 36-66 ) predominantly in the infero-medial portion of the right lower lung zone; a similar but smaller lesion was also seen in the peripheral aspect of the thoracic cavity extending to the lung apex (Fig 2 and 3). The mass was seen causing mediastinal shift supero-lateraly in the ipsilateral hemithorax with mild compression of the contralateral lung tissue. No mediastinal lymphadenopathy, pleural effusion or rib destruction was seen. Abdominal sonograms revealed no abnormality.

An incisional biopsy specimen for histological examination showed blastematous, malignant undifferentiated neoplasm of mesenchymal origin with characteristics of embryonic lung and diagnosis of type III PPB was made. The patient underwent radical surgery. However 24-hours post-op, the respiratory distress worsened with grossly deranged blood gases. In view of severe hypoxia and acidosis he was transferred to the intensive care unit for needful ventilation but he developed sudden cardiac arrest and was certified dead. 


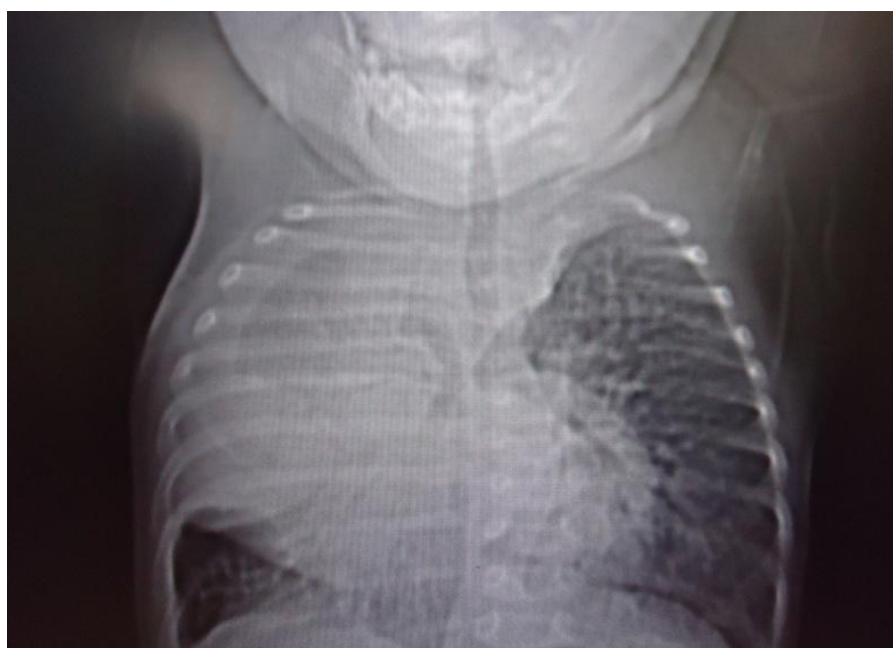

Fig: 1. Frontal chest radiograph shows nearly complete opacity of the right hemithorax and contralateral mediastinal displacement. No rib destruction or pleural fluid collection is seen.

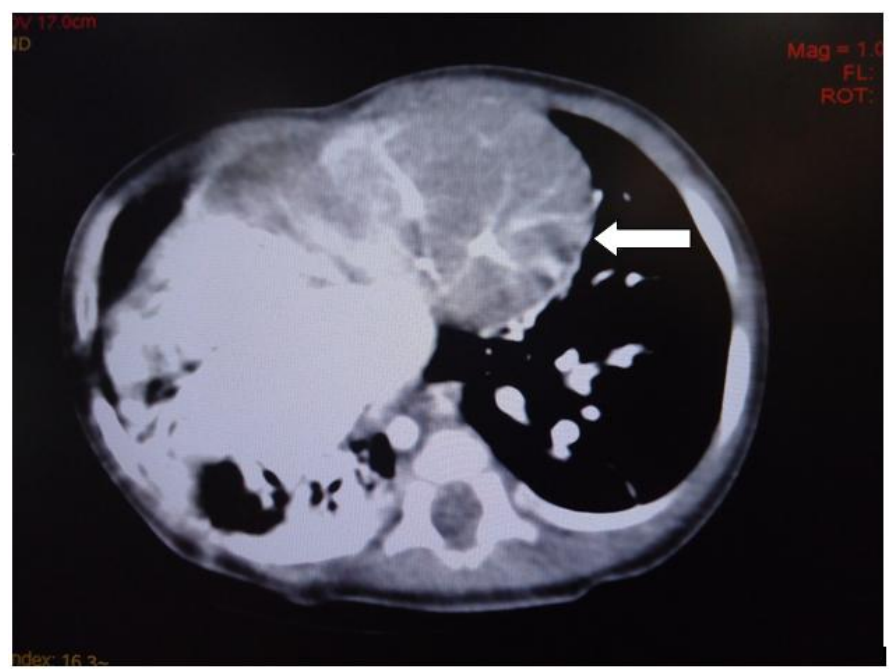

Fig: 2. Contrast enhanced axial CT of the chest (mediastinal window) showing a large right-sided, mildly enhancing, hypodense mass which is occupying the anteromedial portion of the right hemithorax (arrow) with significant mediastinal displacement laterally.

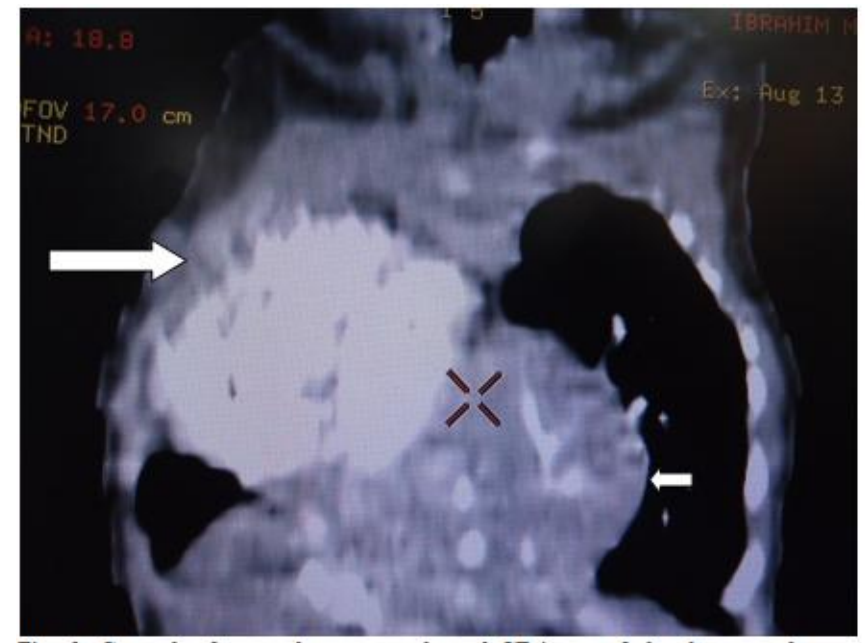

Fig: 3. Coronal reformatted contrast enhanced $\mathrm{CT}$ image of the chest reveals a hypodense, mildly enhancing mass in the infero-medial portion of the right hemithorax (small arrow) displacing the mediastinum superolaterally and compressing the left lung. Lateral chest wall component is also noted (long arrow) . 


\section{DISCUSSION}

Most commonly, lung neoplasms in children are metastatic disease; from Wilm's tumour, osteosarcoma, Ewing's sarcoma and rhabdomyosarcoma. Primary neoplasms of the lung are rare and include papilloma, hamartoma and carcinoids which are benign. Primary malignant neoplasms include various sarcomas and pulmonary blastomas ${ }^{6}$.

Approximately $25 \%$ of cases have been reported ${ }^{7}$, to occur in a familial setting with high prevalence of other tumors, such as multilocular cystic nephromas, thyroid adenomas and carcinomas, sarcomas, medulloblastomas, and germ cell tumors. There was no history of such in any family member of this patient.

The right hemithorax is the most involved in $80 \%$ of reported cases, although bilateral forms have been recently described ${ }^{8}$. In the index case, the tumor was also on the right. Three different types of the lesion have been described on the basis of the histological patterns which are relevant for the prognosis ${ }^{7}$. Type I is mainly cystic, type III mainly solid, whereas type II has solid and cystic components. The three pathologic types are correlated with both age at diagnosis and clinical outcome. Type I occurs in infants (median diagnosis age, 10 months) in contrast to types II and III (median diagnosis ages, 34 and 44 months, respectively) ${ }^{7}$. In the index case, the histologic type was III. Because of the rare occurrence of PPB in childhood, they are usually diagnosed with significant delay, being confused with other neoplasms or pleural empyema ${ }^{1}$.

Delay in the diagnosis due to unspecific symptoms similar to respiratory infections contribute to the poor clinical outcome in general ${ }^{5}$ as in this patient. Imaging is essential in the management of this tumor. Preoperative imaging depicts extension of the tumour into surrounding structures such as the mediastinum, the thoracic wall and the diaphragm, but cannot determine the specific diagnosis. Therefore, diagnosis is based on biopsy of the intrathoracic mass ${ }^{5}$. PPB may be seen on chest radiograph as a well circumscribed mass that often completely fills the affected hemithorax to produce contralateral mediastinal shift. It may be located either peripherally or centrally and there is no definite lobar predominance. It may rarely present as a spontaneouspneumothorax ${ }^{1}$. In the index patient, the radiographic appearance was that of a huge soft tissue dense mass that occupied almost the entire right hemithorax (fig 1) .Radiographic manifestations of 49 patients with pulmonary blastoma reviewed by Koss et $\mathrm{al}^{9}$. consisted of a mass in unilateral lung in 48 patients $(98 \%)$ and multiple nodules in both lungs in one (2\%).

Sonography is superior to radiography in evaluation of the lesion as it shows the tumor as heterogeneously echogenic mass distinct from pleural effusion ${ }^{6}$. Imaging by CT and magnetic resonance imaging(MRI) reveal the exact size of the tumour and are useful for tumor staging.CT appearance of the lesion varies. It may be cystic or partly solid/ partly cystic lesion with a necrotic centre ${ }^{5}$. The mass may have a whorled appearance and display variable contrast enhancement. The presence of pleural or mediastinal involvement is an indication of metastatic disease $^{5}$. In this patient, the mass was solid and showed mild homogenous enhancement. No evidence to suggest mediatinal invasion was seen. Variable intensities of the tumor are seen on MRI depending on the type of lesion (types: I to III) and the imaging sequence employed.They also show enhancement on gadolinium administration ${ }^{5}$.

Positron emission tomography (PET) study is helpful in evaluating tumour vitality and judging the response to neoadjuvant chemotherapy, as well as for decision on the time of surgery. PET reveals pronounced $18 \mathrm{~F}-F D G$ accumulation in the tumor mass indicating vitality ${ }^{5}$. The radiological differential diagnosis for PPB is extensive when the lesion manifests as a solitary pulmonary nodule or mass such as primary or metastatic malignant neoplasm, benign pulmonary parenchymal tumors such as hamartoma, chondroma, and sclerosing hemangioma ${ }^{10}$.Common metastatic sites include the bone, brain, liver, pancreas, kidneys and lymph nodes ${ }^{4}$.

\section{This case showed no evidence of metastasis.}

Treatment of choice for pleuropulmonary blastoma is radical surgery followed by chemotherapy and radiotherapy $^{3}$. The index patient had radical surgey but did not survive long enough for additional treatment options.Five year survival for type II and III lesions is approximately $42 \%$ after multimodality therapy and $83 \%$ for type $\mathrm{I}^{5}$. Patients with pleural, mediastinal and extrapulmonary involvement have a worse prognosis ${ }^{3}$. Local recurrence and the risk of metastases to the lungs, brain, spinal cord and bone occur more often in type II and III $\mathrm{PPB}^{5}$. Neoadjuvant chemotherapy has proved to be effective in the treatment of PPB, resulting in shrinkage of tumour size and allowing complete removal of tumours, giving patients a much better 5 -year survival of $70 \%{ }^{10}$.

The presented case highlights the importance of excluding a malignant cause for an opaque hemithorax in childhood and that PPB should be considered in the differential diagnoses.

\section{References}

[1]. Papaioannou G, Sebire NJ, McHugh K, Imaging of the unusual pediatric blastomas; Cancer Imaging 2009; 9: 1-11.

[2]. Naffaa LN, Donnelly LF. Imaging findings in pleuropulmonary blastoma. Pediatr Radiol. 2005; 35:387-391.

[3]. Lone AR, Wani ML, Wani SN, Ahangar A, Nizami F, Hussain Z et al. Pleuropulmonary blastomain a 3-year-old male child with recurrence in less than two months; Chest Disease Report 2012; 2: 29-30 
[4]. Hashemi A, Souzani A, Souzani A, Keshavarzi S. Pleuropulmonary Blastoma in Children: A Case Report. Iran J Cancer Prev.2012; 5: 105-107.

[5]. Geijer J, Walter K, Uhl M, Bley TA, Juttner E, Brink I et al. Imaging Findings in a 3-year-old Girl with Type III Pleuropulmonary Blastoma; Int J Exp. Clin. pathophysiol. Drg Res. 2007 21: 1119-1122.

[6]. Jobanputra KJ,Talwar IA, Jaggi ST. Pulmonary Blastoma in a Child with Recurrence: A Case Report and Literature Review;Bombay Hosp. J. 2009;51:291-295.

[7]. Tadesse A, Kidane P, Nega B, Schneider J. Pleuropulmonary Blastoma: Case Report; East Cent. Afr. J. Surg. 2013;18:135-138.

[8]. Koss MN, Hochholzer L, O'Leary T. Pulmonary blastomas.Cancer 1991; 67:2368-2381.

[9]. Lee HJ, Goo JM, Kim KW, Im JG, Kim JH. Pulmonary blastoma: radiologic findings in five patients. Clin Imaging2004;28: 113118.

[10]. Kirsch S, Leuschner I, Int-Veen C, Dantonello T, Brecht IB, Schuck A et al: Sixteen children with pleuropulmonary blastoma results of the German cooperative soft tissue sarcoma group. Sarcoma. 2005;9:88 